\title{
Vers un modèle probabiliste pour la formation de réseaux de fissures dans les roches lors de l'injection de $\mathrm{CO}_{2}$
}

M. SEYEDI

BRGM

Service Aménagement et Risques naturels 3. av. Claude Guillemin 45060 Orléans Cedex 2 m.seyedi@brgm.fr

F. HILD

LMT-Cachan, ENS de Cachan CNRS UMR 8535

Université Paris 6

61, av. du Président Wilson 94235 Cachan Cedex
Le stockage géologique de $\mathrm{CO}_{2}$ est une des solutions pour diminuer la concentration de gaz à effets de serre. L'éventuelle fuite de $\mathrm{CO}_{2}$ vers la surface est associée à la perméabilité des roches de couverture et par ce biais peut être reliée à son état de fissuration. Un modèle probabiliste qui prend en compte l'hétérogénéité du matériau est proposé. La propagation de fissures isolées est discutée et la possibilité de formation de réseaux de fissures est analysée. L'interaction entre les fractures est prise en compte en considérant des zones d'obscurcissement correspondant aux effets d'écran créés par chaque fracture.

Mots-clés: stockage géologique de $\mathrm{CO}_{2}$, fracturation des roches, approches probabilistes, modèle de Weibull.

Injection of $\mathrm{CO}$, into geologic formations is being practiced today to decrease the greenhouse gas effect, but it is not yet possible to predict with confidence storage volume, formation integrity and long term reliability. Potential $\mathrm{CO}_{\text {, leakage towards }}$ the surface is associated with the cracking state of caprocks through the permeability-fracturing state relationship. A probabilistic method based on the initial distribution of defects is proposed to describe different aspects of the formation of crack networks in rocks under $\mathrm{CO}$, injection conditions.

Propagation of single cracks is discussed by studying the stress intensity factor variation and the possibility of crack network formation is shown. The interaction between cracks is modelled by considering obscuration zones corresponding to shielding effects created by each crack.

Key words: $\mathrm{CO}_{2}$ geologic disposal, rock fracturing, probabilistic approach, Weibull model. 


\section{Introduction}

Le stockage de $\mathrm{CO}_{2}$ dans les formations géologiques profondes telles que les nappes aquifères salines ou les réservoirs de pétrole déplétés est une des solutions pour diminuer l'effet de la pollution par le $\mathrm{CO}_{2}$ et aussi pour améliorer la rentabilité de la production de pétrole dans le second cas. Actuellement, la stabilité et la sûreté à long terme du stockage de $\mathrm{CO}_{\text {, dans les formations }}$ géologiques profondes sont próbablement le défi le plus important car cet aspect a un impact fort sur l'opinion publique.

La concentration de $\mathrm{CO}_{2}$ en surface peut être très dangereuse (Le Guren et Sigvaldason, 1989; Farrer ef al., 1995; Williams 1995). Le désastre de Lake Nyos en est peut-être l'exemple le plus tristement célèbre. La nuit du 21 août 1986, une masse très importante de $\mathrm{CO}_{2}$ a été émise de Lake Nyos, un lac volcanique au Cameroun. Une concentration mortelle de gaz a été observée à une altitude de $120 \mathrm{~m}$ au-dessus de la surface du lac. La quantité totale de gaz mortel a pu être estimée à $1,3 \mathrm{~km}^{3}$. Le gaz a tué plus de 1700 personnes dans un endroit fortement peuplé et tous les animaux jusqu'à une distance de $14 \mathrm{~km}$. L'hypothèse la plus plausible pour expliquer cette catastrophe est une émission brutale et violente de $\mathrm{CO}_{2}$ causée par la saturation du lac par du $\mathrm{CO}_{2}$ d'origine volcanique (Holloway, 2005). La possibilité d'un accident similaire, causé par une fuite de $\mathrm{CO}_{2}$ à partir d'un réservoir souterrain doit être étudiée. Il faut noter que les particularités topographiques de Lake Nyos ont favorisé la concentration du gaz évacué, ce qui peut donner un caractère singulier à cette tragédie. Pourtant, Cox et al. (1996) ont noté qu'une rupture importante au bord du puits produite vers la fin du processus d'injection, quand la pression dans le réservoir est relativement élevée, peut créer un danger de développement d'un nuage épais de $\mathrm{CO}_{2}$ à la surface.

Des études récentes ont mis en évidence la possibjlité d'apparition d'une rupture mécanique à l'interface réservoir - couverture (Rutqvist et al., 2002 ; Rutqvist et Tsang, 2005). La diminution de la contrainte effective (au sens de la mécanique des sols) dans cette zone peut engendrer la fracturation de la roche. Cette diminution de la contrainte effective est liée à l'augmentation de la pression de la phase fluide (liquide et gazeuse) due à l'injection de $\mathrm{CO}_{2}$. Plusieurs relations existent dans la littérature entre la pression de la phase fluide, $\pi$, et le tenseur de contrainte effective ơ. L'équation (1) en est un exemple (Dangla et Coussy 1998):

$$
\sigma^{\prime}=\sigma-b x I \text { avec } \pi=\left(1-S_{w}\right) \cdot p_{g}+S_{w} \cdot p_{w}-\frac{2}{3} \int_{S w}^{1} p_{c}(\tau) d \tau
$$

où $\mathrm{p}_{\mathrm{g}}$ est la pression de gaz, $\mathrm{p}_{\mathrm{w}}$ la pression de liquide, $\mathrm{p}_{\mathrm{c}}=\mathrm{p}_{\mathrm{g}}-\mathrm{p}_{\mathrm{w}}$ la pression capillaire, et I le tenseur unité. $\sigma$ désigne le tenseur de contrainte totale, $\mathrm{S}_{w}$ la saturation en liquide et b le coefficient de Biot.

L'éventuelle fuite de $\mathrm{CO}_{2}$ vers la surface est associée à la perméabilité du massif rocheux qui entoure le réservoir et par ce biais est reliée à son état de fracturation. Cette influence directe de l'état de fracturation (endommagement) du massif sur la capacité à stocker et la sûreté du réservoir nécessitent la mise en œuvre de modèles appropriés pour modéliser la formation et la propagation de réseaux de fractures dans les massifs rocheux ainsi que l'établissement des relations permet- tant de relier chaque état d'endommagement du massif à sa perméabilité.

L'endommagement par fissuration est le principal processus dissipatif associé au comportement inélastique et à la rupture des roches (quasi) fragiles. Dans ce cas, la déformation irréversible se manifeste par la dégradation progressive du matériau quand les microfissures s'amorcent et se propagent à "petite échelle » et coalescent pour initier des macrofissures à une échelle beaucoup plus grande. Le mécanisme de rupture dans les zones endommagées est de l'amorçage et de la propagation de fissures et de fractures.

Une revue bibliographique révèle une quantité relativement importante de travaux réalisés sur l'endommagement des roches profondes et sur les relations endommagement-perméabilité principalement en vue de la modélisation du stockage profond de déchets nucléaires. Il s'agit principalement d'endommagement sous confinement, vu l'absence de sollicitations en traction et la nature du problème. Des études expérimentales ont montré que différents mécanismes peuvent être à l'origine de l'amorçage et de la propagation de fractures sous des contraintes de compression (Souley et al., 2001). Les mécanismes concernés incluent le glissement le long des faces de fissures préexistantes ou de grains, l'écrasement de pores, le mouvement de dislocations, etc. Afin de décrire ces mécanismes, plusieurs modèles micromécaniques de rupture ont été développés. La caractéristique principale des modèles micromécaniques est leur capacité à décrire l'évolution des microfissures au niveau de la microstructure de la roche.

Par ailleurs, des modèles anisotropes d'endommagement ont aussi été développés. Ils sont basés sur des principes thermodynamiques et utilisent une variable interne pour caractériser l'état d'endommagement de la roche. En effet, l'amorçage de microfissures affecte les propriétés mécaniques du matériau. Différentes méthodes ont été développées afin d'évaluer des paramètres élastiques d'un solide fissuré. Parmi cellesci, les modèles dits « autocohérents » et le schéma différentiel (Budiansky et O'Connell, 1976; Hashin, 1988) sont les plus utilisés. Les principaux ingrédients de chaque modèle micromécanique sont le critère d'amorçage des fissures, les lois de propagation et l'évaluation de l'effet de la création de microfissures sur la souplesse du matériau. L'avantage essentiel de ces modèles est leur capacité à décrire l'amorçage et la propagation de microfissures à une échelle appropriée. Cependant, l'implantation numérique de ces modèles n'est pas toujours facile surtout pour des problèmes 3D.

Récemment une troisième voie, que l'on peut appeler « approche phénoménologique enrichie », a été développée (Halm et Dragon, 1996 ; Homand-Étienne et al., 1998: Hoxa et Homand, 1999). Des approches essentiellement basées sur les travaux de Kachanov (1994) peuvent également ètre utilisées pour calculer des propriétés effectives d'un solide fissuré. Ainsi, un modèle anisotrope a été proposé par Shao et al. (1999) pour décrire l'endommagement dans les roches.

D'un point de vue pratique, l'étude de la sûreté d'un stockage géologique de $\mathrm{CO}_{2}$ consiste à déterminer les marges de sécurité dont on dispose pour une pression d'injection donnée. Ceci entraîne inévitablement la détermination de la pression limite pour chaque site de stockage. Cette pression limite, d'une part, doit être assez élevée pour permettre l'injection de quantités importantes 
de gaz et, d'autre part, ne pas mettre en périll'intégrité de la roche de couverture. La prise en compte de l'hétérogénéité de la roche avec en particulier la présence de microfractures et de fissures, est un point important dans la démarche adoptée dans le présent travail pour déterminer cette pression limite. Pour ce faire, nous nous sommes limités, dans le cadre de cet article, à l'étude de la formation de réseaux de fractures en mode I, c'est-àdire la rupture en mode d'ouverture.

Il est important de noter qu'une étude complète de l'intégrité d'un stockage géologique de $\mathrm{CO}_{2}$ doit prendre en compte les effets des couplages hydromécaniques, les variations de température et des processus géochimiques sur le comportement des formations de couverture. Dans le cadre de cet article, la formation des réseaux de fractures dans la roche de couverture, considérée comme un matériau hétérogène, sous le seul effet mécanique de la surpression engendrée par l'injection de gaz, est étudiée.

La rupture de la roche est un phénomène généralement aléatoire. La contrainte de rupture présente une dispersion et sa valeur moyenne diminue quand le volume de l'échantillon augmente. L'effet de cette dispersion sur la capacité du site à stocker le gaz doit être pris en compte. Les modèles probabilistes permettent de prendre en compte l'effet de l'hétérogénéité de la roche sur son état d'endommagement. L'hétérogénéité du massif rocheux est représentée par la présence de fissures initiales réparties de manière aléatoire dans le massif. Ces dernières sont à l'origine de l'amorçage de fissures entrainant la rupture. Un modèle probabiliste basé sur un processus ponctuel de Poisson de microfissures (Gulino et Phoenix, 1991; Jeulin, 1991), sites potentiels d'amorçage des fissures, est utilisé pour décrire ce caractère aléatoire en reliant la microstructure du matériau à son comportement macroscopique.

Les différents aspects de la propagation de fissures isolées sont étudiés, et la possibilité d'arrêt est évaluée. La formation de réseaux de fissures est ensuite traitée. L'amorçage de chaque fissure modifie le champ de contraintes dans son voisinage, et des zones de relaxation et d'amplification de contraintes sont créées. L'effet d'écran peut être expliqué en considérant l'influence des zones de relaxation de contraintes sur l'amorçage d'autres fissures. L'éventuelle interaction entre les fissures amorcées et les autres sites d'amorçage est modélisée en introduisant une probabilité d'écrantage pour chaque site d'amorçage.

\section{2}

\section{Conditions d'amorçage de fissures}

\section{1}

\section{Rappel de mécanique de la rupture}

La mécanique de la rupture propose une solution pour estimer la valeur des paramètres qui gouvernent le comportement des fissures, c'est-à-dire leur amorçage, propagation et arrêt. Trois types de variables caractérisent la perturbation du champ de contraintes due à la présence d'une fissure: les facteurs d'intensité des contraintes, les intégrales de contour et le taux de restitution d'énergie. Considérant la commodité de calcul du facteur d'intensité des contraintes $\mathrm{K}$, nous l'utilisons, dans le cadre du présent travail, pour étudier le comportement des fractures dans un massif rocheux.
Les facteurs d'intensité des contraintes caractérisent l'intensité du champ des contraintes à la pointe de la fissure. Si I'on connait leur valeur, ils permettent de déterminer complètement les champs de contraintes singuliers ou de déplacements associés dans la structure fissurée, considérée comme élastique. Inversement, si l'on connaît les expressions des composantes non nulles des contraintes et des déplacements, on peut déterminer les facteurs d'intensité des contraintes pour les trois modes d'ouverture de fissure (Fig. 1a) par les expressions suivantes qui sont valables pour les milieux fissurés plans :

$$
\begin{gathered}
K_{I}=\operatorname{Lim}_{r \rightarrow 0}\left(\sigma_{22} \sqrt{2 \pi r}\right)=\lim _{r \rightarrow 0}\left(\frac{E}{8 H_{v}} \sqrt{\frac{2 \pi}{r}}\left[u_{2}\right]\right) \\
K_{I I}=\operatorname{Lim}_{r \rightarrow 0}\left(\sigma_{12} \sqrt{2 \pi r}\right)=\lim _{r \rightarrow 0}\left(\frac{E}{8 H_{v}} \sqrt{\frac{2 \pi}{r}}\left[u_{1}\right]\right) \\
K_{\text {III }}=\operatorname{Lim}_{r \rightarrow 0}\left(\sigma_{13} \sqrt{2 \pi r}\right)=\lim _{r \rightarrow 0}\left(\frac{E}{8(1+v)} \sqrt{\frac{2 \pi}{r}}\left[u_{3}\right]\right)
\end{gathered}
$$

avec $\mathrm{H}_{v}=1$ en contraintes planes et $\mathrm{H}_{v}=1-\mathrm{v}^{2}$ en déformations planes. Dans l'équation (2), $K_{1}, K_{11}$ et $K_{\text {III }}$ sont les facteurs d'intensité des contraintes (FIC), E le module d'élasticité, $v$ le coefficient de Poisson, [u] la discontinuité de champ de déplacements selon l'axe i et $r$ la distance entre chaque point et la pointe de fissure (Fig. 1b).

Dans la suite, nous ne considérons que le mode I d'ouverture et le facteur d'intensité des contraintes est considéré comme le pilote d'amorçage et de propagation des fissures.

\section{2}

\section{Hypothèses}

Deux phases peuvent être distinguées lors d’un processus de stockage de $\mathrm{CO}_{2}$. Pendant la phase d'injection, la pression du gaz augmente et atteint sa valeur maximale à la fin de l'injection. En négligeant la fuite diffuse de gaz et sa dissolution dans la phase liquide sur une échelle de ternps relativement courte, on peut considérer que la pression reste constante pendant la seconde phase, c'est-à-dire le stockage. La question que l'on se pose est d'étudier l'effet de cette augmentation de pression sur l'équilibre mécanique du massif et de savoir comment les réseaux de fractures peuvent évoluer suite à l'injection de gaz.

Afin d'expliquer la formation de fissures, on suppose que toutes les fissures s'amorcent sur des défauts préexistants, modélisés par des fissures possédant une taille donnée. Autrement dit, le début de la propagation est considéré comme l'amorçage. Dans ce cadre, l'amorçage de fissures est limité à la phase d'injection et aucune fissure ne pourra s'amorcer pendant la seconde phase. Toutefois, des fissures amorcées peuvent continuer à se propager.

Le modèle de Weibull est largement utilisé pour étudier la dégradation et la rupture des matériaux à comportement fragile (Weibull, 1939) ou quasi fragile (Daniels, 1944; Coleman 1958; Hild et al., 1994). Lors de la rupture d'une éprouvette de roche, on peut considérer que c'est la rupture du défaut le plus critique, la rupture locale, qui entraîne la rupture de la structure, donc la rupture globale. Autrement dit, la rupture du maillon le plus faible entraine la rupture de la chaine 

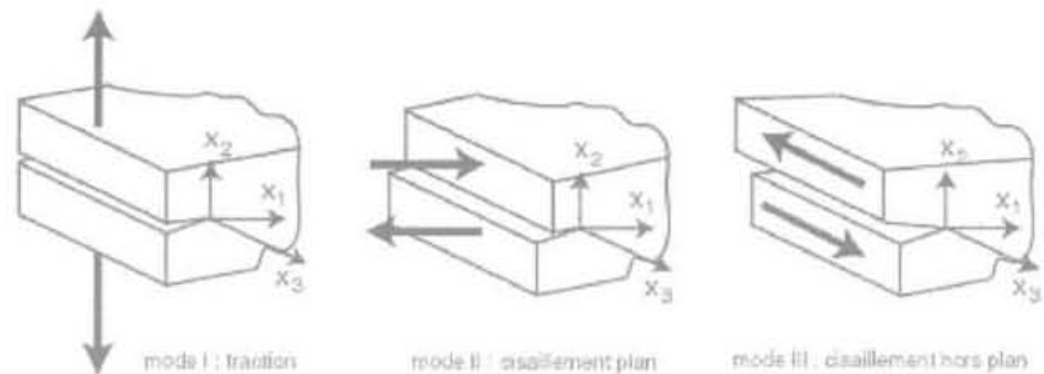

mode il ' asolstmentpian

mode fll cinaillement aors plan

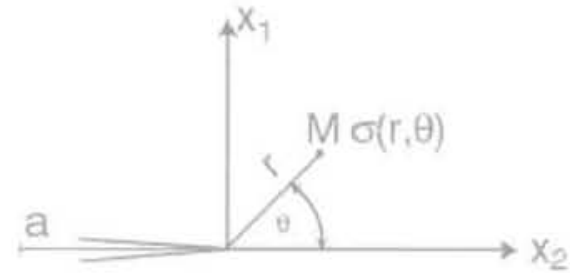

FIG. 1 a) Modes de sollicitations d'une fissure; b) repères locaux à la pointe d'une fissure (Lemaitre et Chaboche 1985).

a) Opening modes for a crack. (b) local coordinate systems in the vicinity of a crack tip (Lemaitre and Chaboche, 1985).

(la structure). Dans le cadre de l'hypothèse du maillon le plus faible (Freudenthal, 1968), la probabilité de rupture globale $\mathrm{P}_{\mathrm{F}}$ d'un domaine $\Omega$ est reliée à la probabilité de chaque élément $\Omega_{0}$ de surface $\mathrm{S}_{0}, \mathrm{P}_{\mathrm{Fo}}$ par :

$$
P_{F}=1-\exp \left[\frac{1}{S_{0}} \int_{\Omega} \ln \left(1-P_{F 0}\right) d \Omega\right]
$$

La probabilité cumulée de la rupture locale $\mathrm{P}_{\mathrm{F} 0}$ peut être écrite à partir d'une loi de Weibull (1951) :

$$
\ln \left(1-P_{F 0}\right)=-\left(\frac{\left\langle\sigma_{1}-\sigma_{u}\right\rangle}{\sigma_{0}}\right)^{m}
$$

où $\sigma_{1}$ est la contrainte principale maximale dans l'élément considéré, $\langle x\rangle$ la partie positive de $x(\langle x\rangle=x$ si $x \geq 0$ et $\langle x\rangle=0$ sinon), $m$ le paramètre de forme (paramètre de Weibull), $S_{0} \sigma_{0}^{m}$ le paramètre d'échelle et $\sigma_{u}$ un paramètre seuil. Si l'on prend $\sigma_{u}=0$, les équations (3) et (4) représentent un modèle de Weibull à deux paramètres. D'un point de vue physique, $P_{\text {mi }}$ représente la probabilité de trouver un défaut critique dans un domaine $\Omega_{0}$. Cette probabilité est reliée à une distribution de défauts caractérisée par une densité de probabilité $\mathrm{f}_{0}$. La distribution initiale de défauts, $\mathrm{f}_{0}$, đépend de paramètres morphologiques du défaut ; tout d'abord sa taille a, ensuite son orientation qui peut être définie par un vecteur normal unitaire $\underline{n}$ et enfin d'autres paramètres morphologiques qui sont décrits par w. Pour un niveau de chargement donné, la population de défauts existants peut ètre séparée en deux parties. La première, $\mathrm{D}$, contient les défauts critiques. La seconde, $\mathrm{D}_{\mathrm{n}}$ contient les défauts qui ne sont pas critiques pour ce niveau de contrainte. La probabilité cumulée de la rupture $\mathrm{P}_{\mathrm{F}}(\mathrm{Q})$ pour un chargement de niveau Q s'écrit (Hild et Marquis, 1995) :

$$
P_{F 0}(Q)=\int_{D(Q)} f_{0}(a, n, w) d a d n d w
$$

En utilisant cette définition, la valeur de $\mathrm{P}_{\mathrm{FO}}$ peut varier entre $0\left(D_{-}=\emptyset\right.$ : aucun défaut critique) et $1\left(D_{c}=\right.$ $\mathrm{D}$ : tous les défauts sont critiques). Dans le cadre de la présente étude, tous les défauts sont considérés comme des fissures. Chacun de ces défauts peut commencer à se propager si sa valeur correspondante de FIC dépasse la ténacité de la roche.

En supposant que l'hypothèse du maillon le plus faible (Freudenthal, 1968) peut être appliquée à la rupture de roches, un modèle de Weibull (1951) à deux paramètres peut être utilisé pour représenter la dispersion des résultats expérimentaux et l'équation (4) s'écrit dans le cas d'un problème plan:

$$
P_{\mathrm{F}}=1-\exp \left[-\left(\frac{\mathrm{H}_{m} \mathrm{~S}}{\mathrm{~S}_{0}}\right)\left(\frac{\sigma_{F}}{\sigma_{0}}\right)^{\mathrm{m}}\right]
$$

où $\mathrm{P}_{\mathrm{F}}$ est la probabilité de rupture, $\mathrm{H}_{\mathrm{m}}$ le facteur d'hétérogénéité des contraintes (Hild et aĺ, 1992) dont l'expression sera précisée plus bas, S la surface sollicitẻe, $S_{0} \sigma_{0}^{m}$ le paramètre d'échelle de Weibull, $\sigma_{-}$la contrainte à rupture et m le module de Weibull. Mathématiquement, le modèle de Weibull peut être décrit par un processus ponctuel de Poisson (Gulino et Phoenix, 1991 ; Jeulin, 1991) d'intensité $\lambda_{r}\left(\sigma_{1}\right)$ :

$$
\lambda_{t}(\sigma)=\frac{1}{S_{0}}\left(\frac{\langle\sigma\rangle}{\sigma_{0}}\right)^{m}
$$

où $\lambda$ est la densité des défauts activables, $m$ le module de Weibull, $<\bullet>$ la partie positive et $\sigma_{0}$ un paramètre d'échelle lié à une surface de référence $S_{j}$. La probabilité $\mathrm{P}$ de trouver $\mathrm{N}=\mathrm{n}$ défauts activables dans un domaine $\Omega$ de surface $\mathrm{S}$ soumis à un chargement uniforme suit une loi de Poisson :

$$
P(N=n)=\frac{\left[\lambda_{1}\left(\sigma_{1}\right) S\right]^{n}}{n !} \exp \left[-\lambda_{1}(\sigma) S\right]
$$

En faisant 1'hypothèse du maillon le plus faible, la probabilité de rupture $\mathrm{P}_{\mathrm{f}}$ est égale à la probabilité de trouver au moins un défaut critique dans un domaine $\Omega$ de surface S (Hild, 2001) :

$$
P_{F}=P(N \geq 1)=1-P(N=0)=1-\exp \left[-\frac{S}{S_{0}}\left(\frac{\langle\sigma\rangle}{S_{0}}\right)^{m}\right]
$$


L'équation (9) est identique aux équations (3) et (4) pour un domaine $\Omega$ soumis au chargement uniforme. Notons que dans le cadre du modèle de Poisson-Weibull, les sites potentiels de rupture sont considérés comme des points avec une répartition aléatoire. C'est ce modèle qui sert de base à la description de la formation de réseau ; les paramètres pouvant, par exemple, être identifiés à partir d'essais où l'hypothèse du maillon le plus faible s'applique (Denoual et Hild, 2000).

Pour une fissure donnée de la taille a et en mode I, l'expression du facteur d'intensité des contraintes (équation (1)) peut étre réécrite de la manière suivante :

$$
K=Y \sigma \sqrt{a}
$$

où Y est un paramètre adimensionnel et a la contrainte appliquée. Une fissure peut commencer à se propager, une fois que $\mathrm{K}$ dépasse la valeur de la ténacité de la roche $\mathrm{K}_{\mathrm{IC}}$. La contrainte d'amorçage $\sigma_{1}$ de chaque fissure peut être donc reliée à sa taille par:

$$
\sigma_{i}=\frac{K_{1 c}}{Y \sqrt{a}}
$$

La réponse mécanique à l'échelle du réservoir n'est pas le sujet du présent travail. Afin de pouvoir analyser les conditions d'amorçage de fissures au bord des puits d'injection et dans la couverture par des solutions analytiques, on idéalise leur géométrie par un cylindre creux soumis à une pression interne (Fig. 2). Dans cette modélisation, la couverture est considérée comme un cylindre entourant le réservoir. L'effet de la pesanteur n'étant pas pris en compte, la modélisation ne considère que la traction engendrée dans la couverture par l'injection de gaz. Dans ce cadre, la surpression engendrée par l'injection de gaz est modélisée comme une pression appliquée sur la surface interne du cylindre, c'est-à-dire sur l'interface réservoir - couverture. Dans ce cas le champ des contraintes s'écrit :

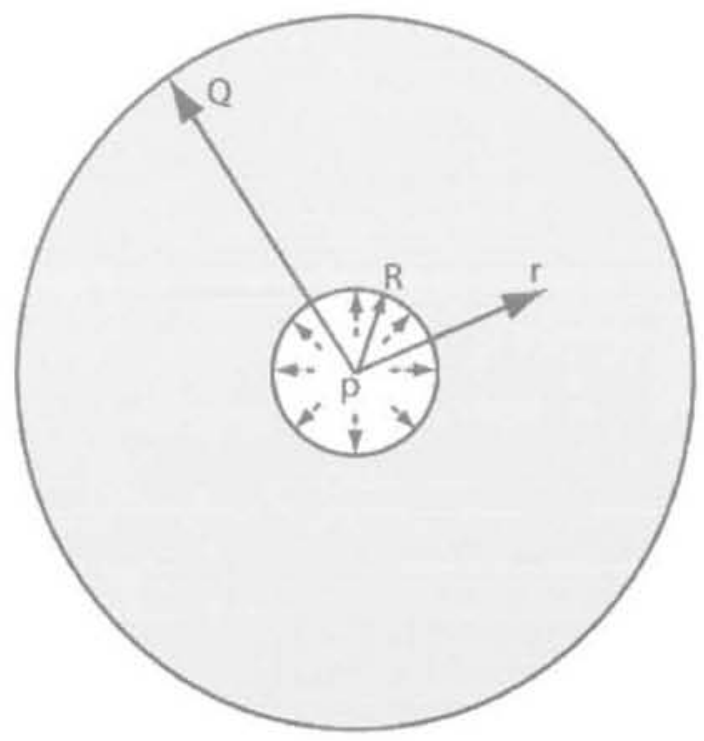

FIG. \& Réservoir ou puits d'injection idéalisés par un cylindre creux sous pression interne. Reservoir or injection well idealized as a hollow cylinder under internal pressure $\mathrm{p}_{\text {int }}$.

$$
\begin{gathered}
\sigma_{\pi t}=\frac{p_{i n t} R^{2}}{Q^{2}-R^{2}}\left(1-\frac{Q^{2}}{r^{2}}\right) \\
\sigma_{\theta \theta}=\frac{p_{i n t} R^{2}}{Q^{2}-R^{2}}\left(1+\frac{Q^{2}}{r^{2}}\right) \\
\sigma_{z z}=2 v \frac{p_{i n t} R^{2}}{Q^{2}-R^{2}}
\end{gathered}
$$

où $\mathrm{p}_{\text {int }}$ est la pression interne, $\mathrm{R}$ et $\mathrm{Q}$ les rayons interne et externe, $\mathrm{r}$ la coordonnée radiale et $v$ le coefficient de Poisson. Connaissant le champ de contrainte, on peut calculer le facteur d'hétérogénéité des contraintes $\mathrm{H}_{\mathrm{m}}$. Ce dernier caractérise l'effet de l'hêtérogénéité du champ des contraintes (Hild et al., 1992) sur la probabilité cumulée de la rupture $\left(\mathrm{H}_{\mathrm{m}}=1\right.$ quand un domaine est sollicité en traction pure). En considérant que la contrainte orthoradiale est la principale responsable de la fissuration (car la seule positive, en traction), $\mathrm{H}_{\mathrm{m}}$ est calculé de la manière suivante :

$$
H_{m}=\frac{1}{S} \int\left(\frac{\sigma_{\theta \theta}(r)}{\sigma_{F}}\right)^{m} d \Omega
$$

où $\mathrm{d} \Omega=$ rdrd $\theta$. L'équation (13) peut être intégrée en la développant en série limitée. Quand m est un nombre entier on a :

$H_{m}=\frac{1}{\pi\left(Q^{2}-R^{2}\right)}\left(\frac{1}{1+(Q / R)^{2}}\right)^{m} \iint_{0}^{2 \pi} \sum_{i=0}^{m} \alpha_{i}\left(\frac{Q^{2}}{r^{2}}\right)^{i} r d r d \theta(14)$

où $\alpha$ est le coefficient du développement en série et s'écrit:

$$
\alpha_{i}=\frac{m !}{(m-i) ! i !}
$$

Les valeurs de $\mathrm{H}_{\mathrm{m}}$ en fonction de $\mathrm{m}$ et du rapport Q/R sont présentées sur la figure 3. Ces résultats montrent que lorsque $Q / R$ tend vers $1, H_{m}$ tend aussi vers 1

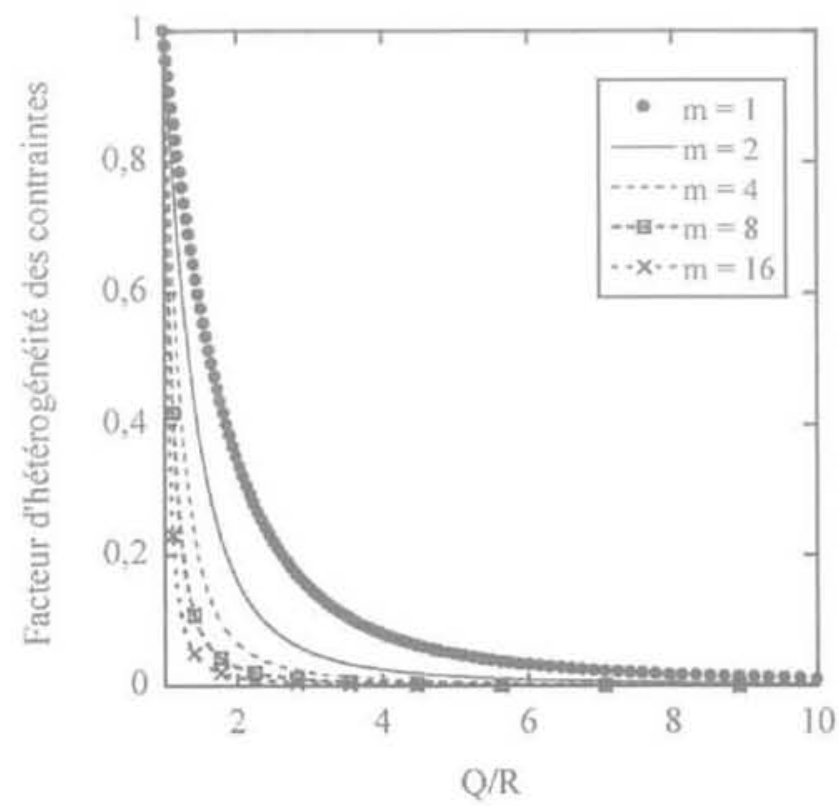

FG. 3 Facteur d'hétérogénéité des contraintes en fonction du module de Weibull $\mathrm{m}$ et $\mathrm{du}$ rapport de rayons $Q / R$.

Stress heterogeneity factor as a function of $\mathrm{Q} / \mathrm{B}$ for different Weibull moduli m. 
et un champ de contraintes quasi uniforme existe. Par contre, quand $\mathrm{Q} / \mathrm{R}$ augmente, le champ de contraintes devient plus hétérogène et la valeur de $\mathrm{H}_{m}$ diminue. Cette tendance s'amplifie quand $\mathrm{m}$ augmente.

\section{3}

\section{Probabilite d'amorçage de fissures}

L'équation (6) définit la probabilité d'amorçage quand l'hypothèse du maillon le plus faible est applicable. Si l'on remplace la valeur de $\mathrm{H}_{\mathrm{m}}$ dans l'équation (6) par sa définition, on obtient la probabilité d'amorçage pour différents rapports $Q / R$ et $S / S_{0}$ en fonction de la contrainte de rupture normée (Fig. 4). Pour une valeur donnée de m, $\mathrm{H}_{\mathrm{m}}$ diminue quand $\mathrm{Q} / \mathrm{R}$ augmente. $\mathrm{Par}$ conséquent, pour le même niveau de pression interne, la probabilité d'amorçage est plus faible pour les grands rapports Q/R. Quand $\mathrm{H}_{\mathrm{m}}$ est proche de 1, tous les points sont sollicités de la même manière. Quand Q/R augmente, le champ de contraintes devient plus hétérogène, la probabilité de rupture diminue. Ce résultat met en évidence l'effet de l'épaisseur de la couverture par rapport à celui du réservoir sur le risque de fracturation de la couverture. L'augmentation de l'épaisseur de la couverture par rapport au réservoir diminue le risque de sa fracturation.

La figure $4 \mathrm{~b}$ montre la variation de la probabilité de rupture en fonction de la contrainte de rupture pour différentes valeurs de $\mathrm{S} / \mathrm{S}_{0}$. Ce graphique illustre l'effet d'échelle sur la probabilité d'amorçage. Pour le même niveau de la pression, la probabilité d'amorçage augmente quand la surface sollicitée augmente.

\section{3}

\section{Formation de réseaux de fissures}

Dans ce paragraphe, on étudie tout d'abord la propagation d'une fissure isolée dans l'épaisseur du cylindre. Par cette analyse, on démontre qu'un réseau de fissures peut être formé suite à l'application d'une pression interne, si une fissure peut s'amorcer. Ensuite, un modèle probabiliste est introduit pour expliquer la formation de ces réseaux de fissures. Il est basé sur une extension du modèle de Poisson-Weibull introduit cidessus. En effet, il s'agira de prendre en compte l'interaction de fissures s'étant propagé avec des sites potentiels d'amorçage de nouvelles fissures.

\section{3.}

\section{Propagation d'une fissure isolée}

Considérons une fissure débouchante sur le bord intérieur d'un cylindre creux soumis à une pression interne $\mathrm{p}_{\text {int. }}$ Le facteur d'intensité des contraintes est considéré comme le paramètre clé qui pilote la propagation de cette fissure. Pour étudier si une fissure se propage ou pas suite à une augmentation de $\mathrm{p}_{\text {. }}$ la valeur de $\mathrm{K}$ est comparée à la ténacité de la roche $\mathrm{K}_{1 \mathrm{c}}$ pour différentes longueurs de fissure quand la pression intérieure augmente. Pour ce faire, des simulations par éléments finis ont été réalisées dont les résultats sont présentés sur la figure 5 . Ces courbes montrent deux tendances différentes pour la variation du facteur d'intensité des contraintes en fonction de la taille de la fissure. Dans la première partie, l'effet de la taille de fis-

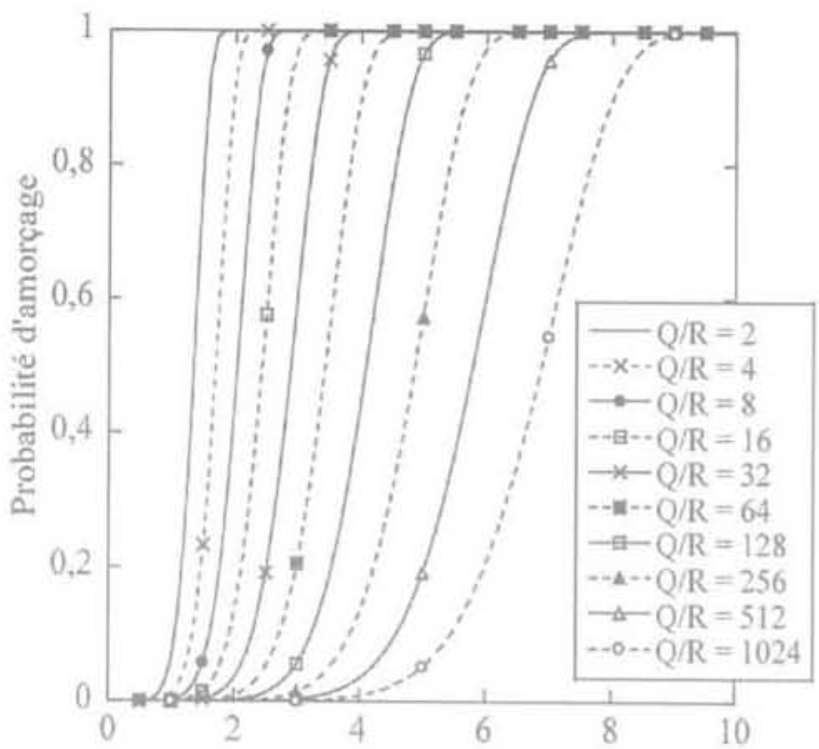

Contrainte de la rupture normée $\left(\sigma_{\mathrm{F}} / \sigma_{0}\right)$

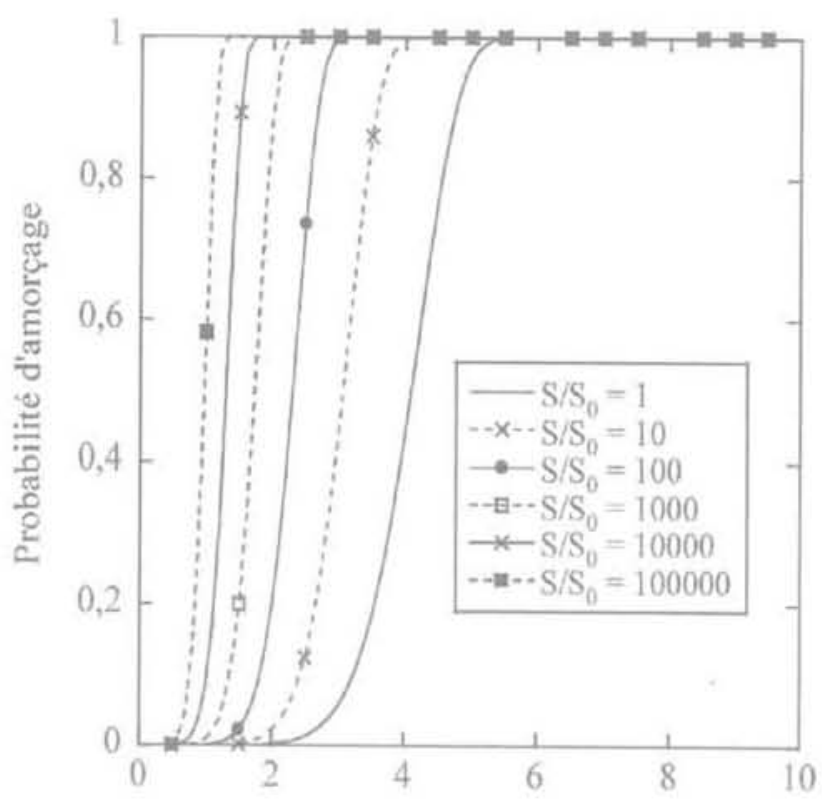

Contrainte de la rupture normée $\left(\sigma_{p} / \sigma_{0}\right)$

FIG. 4 a) Effet de $Q / R$ sur la probabilité d'amorçage; b) effet de $S / S_{0}$ sur la probabilité de rupture.

a) Effect of the $Q / \mathrm{R}$ ratio on the initiation probability as a function of the normalized failure stress: b) effect of the $S / S_{0}$ ratio on the initiation probability as a function of the normalized failure stress.

sure par rapport au niveau de la contrainte appliquée est prépondérant et $\mathrm{K}$ augmente avec celle-ci. Par contre dans la seconde partie, l'effet de la diminution du niveau de contrainte appliquée à la fissure devient plus important que l'effet de l'augmentation de sa taille et $\mathrm{K}$ diminue en fonction de la taille de fissure. La figure 5 montre qu'une fissure peut s'amorcer dès lors que $\mathrm{K}$ atteint la ténacité $\left(\mathrm{K}_{1 \mathrm{c}}\right)$. La fissure se propage en augmentant la pression interne. Quand la pression intérieure arrive à sa valeur maximale, la fissure continue à se propager et s'arrête lorsque la valeur de $\mathrm{K}$ devient inférieure à $\mathrm{K}_{1 \mathrm{c}}$. 


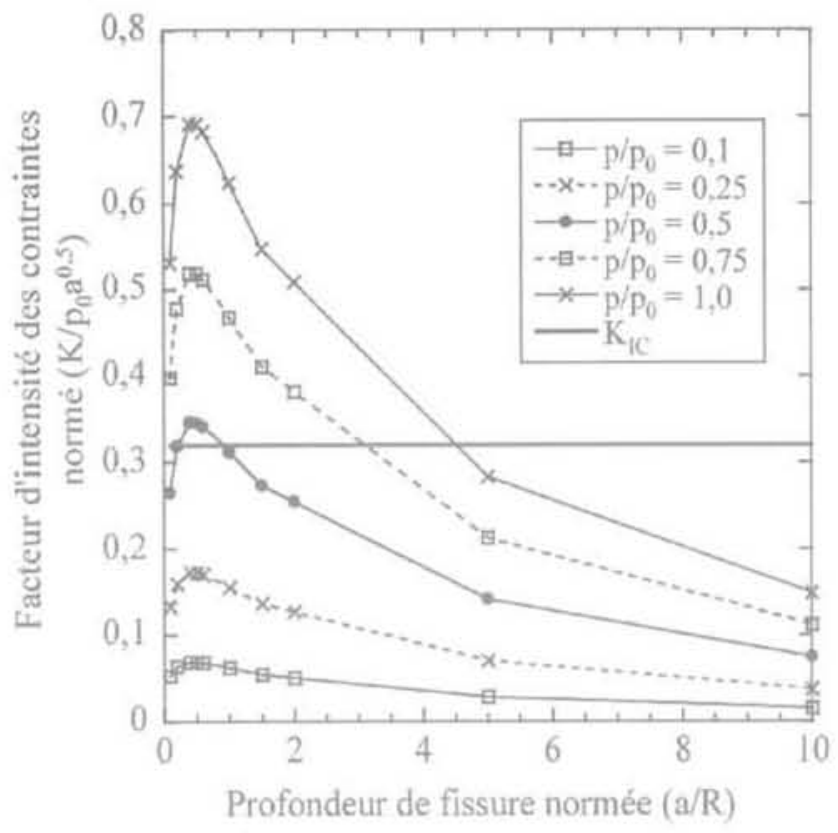

FIG.5 Étude de la propagation d'une fissure isolée : comparaison de $\mathrm{K}$ et $\mathrm{K}_{1 \mathrm{c}}$.

Stress intensity factor versus dimensionless crack size for different applied pressures $p_{m i}$. The fracture toughness is also depicted to show crack arrest.

Ces résultats montrent qu'une fissure isolée se propage jusqu'à une taille donnée pour laquelle la valeur du facteur d'intensité des contraintes devient inférieure à la ténacité de la roche. Quand une première fissure s'arrête, une autre peut s'amorcer sur un deuxième site d'amorçage. La deuxième fissure s'arrêtera à la même longueur que la première, une troisième fissure peut s'amorcer et ainsi de suite. Cela signifie qu'un réseau de fissures peut se former.

\section{9}

\section{Modélisation probabiliste de la formation de réseaux de fissures}

Supposons que toutes les fissures s'amorcent à partir de défauts initiaux répartis de manière aléatoire dans le massif. Un processus ponctuel de Poisson est considéré pour décrire la répartition des sites d'amorçage (\$2.2). Parmi tous ces sites potentiels d'amorçage de fissure, seule une fraction va donner naissance à des fissures. Quand la valeur du facteur d'intensité des contraintes pour un défaut (une fissure) dépasse une valeur critique $\left(\mathrm{K}_{1}\right)$, la fissure commence à se propager (voir équation (11)). Quand une fissure est initiée, la présence de cette fissure crée une zone de relaxation de contraintes autour de celle-ci. Pour expliquer l'amorçage d'une fissure, il faut modéliser l'interaction des zones (donc le volume, la surface ou la longueur) affectées par la diminution de contrainte et les autres sites qui peuvent créer des fissures. Si le site se trouve dans une zone de relaxation des contraintes, la contrainte microscopique est inférieure à la contrainte macroscopique appliquée. Dans ce cas, ce site ne peut pas donner naissance à une nouvelle fissure.

Dans cette approche, la zone d'obscurcissement est la quantité-clé pour décrire la formation d'un réseau de fissures. La zone d'obscurcissement est définie comme une zone dans laquelle le niveau de contrainte est inférieur à la contrainte appliquée. Une série de simulations par éléments finis a été réalisée pour différentes configurations géométriques afin d'obtenir une estimation de la taille de cette zone au voisinage d'une fissure amorcée sur le bord d'un cylindre creux soumis à une pression interne. La forme de cette zone est représentée pour trois rapports différents de taille de fissures par rapport au rayon interne du cylindre sur la figure 6. Ces résultats montrent que la taille de la zone d'obscurcissement est proportionnelle à la taille de la fissure, elle-même proportionnelle à la pression interne imposée p et une fonction puissance est utilisée pour la représenter en première approximation :

$$
\mathrm{Z}_{\mathrm{obs}}(\mathrm{p})=\left(\frac{\mathrm{Ap}}{\sigma_{0}}\right)^{\mathrm{n}}
$$

où $Z_{\text {obs }}$ est la taille de la zone d'occultation, A une constante et $n$ la dimension de l'espace $(n=1,2$ ou 3). La distribution de sites est divisée en deux parties et la densité moyenne de fissures initiées s'écrit :

$$
\lambda_{\mathrm{b}}(\mathrm{p})=\lambda_{4}(\mathrm{p})-\lambda_{\mathrm{obs}}(\mathrm{p})
$$

où $Z \lambda_{b}(p)$ donne le nombre moyen de fissures dans une zone de mesure $Z$ pour une pression appliquée inférieure ou égale à p. Les indices désignent la densité de fissuration (b), la densité de défauts écrantés (obs) et la densité totale de défauts activables (t).

Deux conditions doivent être satisfaites pour l'amorçage de nouvelles fissures. Il faut qu'un site se trouve dans la zone étudiée et que celui-ci ne soit écranté par aucune fissure. L'incrément de densité de fissures initiées s'écrit:

$$
\frac{d \lambda_{b}}{d p}(p)=\frac{d \lambda_{1}}{d p}(p)\left[1-P_{o b s}(p)\right] \operatorname{avec} \lambda_{b}(0)=0
$$

où $1-\mathrm{P}_{\text {os }}$ est la probabilité pour qu'aucune fissure n'écrante le site. La variable $1-\mathrm{P}_{\text {obs }}$ peut ètre divisée en une infinité d'événements définis par la probabilité de ne pas trouver de défaut dans la zone d'occultation $Z_{\text {obss }}(p)$ pour un niveau de pression p pendant un incrément de pression dp. Cet incrément de probabilité est décrit par un processus ponctuel de Poisson d'intensité $\mathrm{d} \lambda / \mathrm{dp}$. Ces événements indépendants sont utilisés pour calculer $P_{\text {obs }}$ :

$$
P_{\text {obs }}\left(P_{\text {int }}\right)=1-\exp \left[-\int_{0}^{P_{\text {int }}} \frac{d \lambda_{1}}{d p}(p) Z_{\text {obs }}(p) d p\right]
$$

On notera que la probabilité d'obscurcissement permet de définir l'état d'endommagement de la structure considéré. En effet, la fraction volumique des zones obscurcies (donc endommagée) est directement égale à cette quantité. Ceci permet de définir une variable d'endommagement (Kachanov, 1958; Lemaitre et Chaboche, 1978) associée au réseau étudié. De ce point de vue, on se rapproche d'études de matériaux à comportement quasi fragile (Daniels, 1944 ; Coleman, 1958 ; Hild et al., 1994).

Au début du chargement, il y a peu d'interaction entre les fissures créées et $\lambda_{\mathrm{b}}(\mathrm{p})=\lambda_{\mathrm{v}}(\mathrm{p})$, mais au fur et à mesure que davantage de fissures s'amorcent, l'interaction entre les fissures devient de plus en plus 

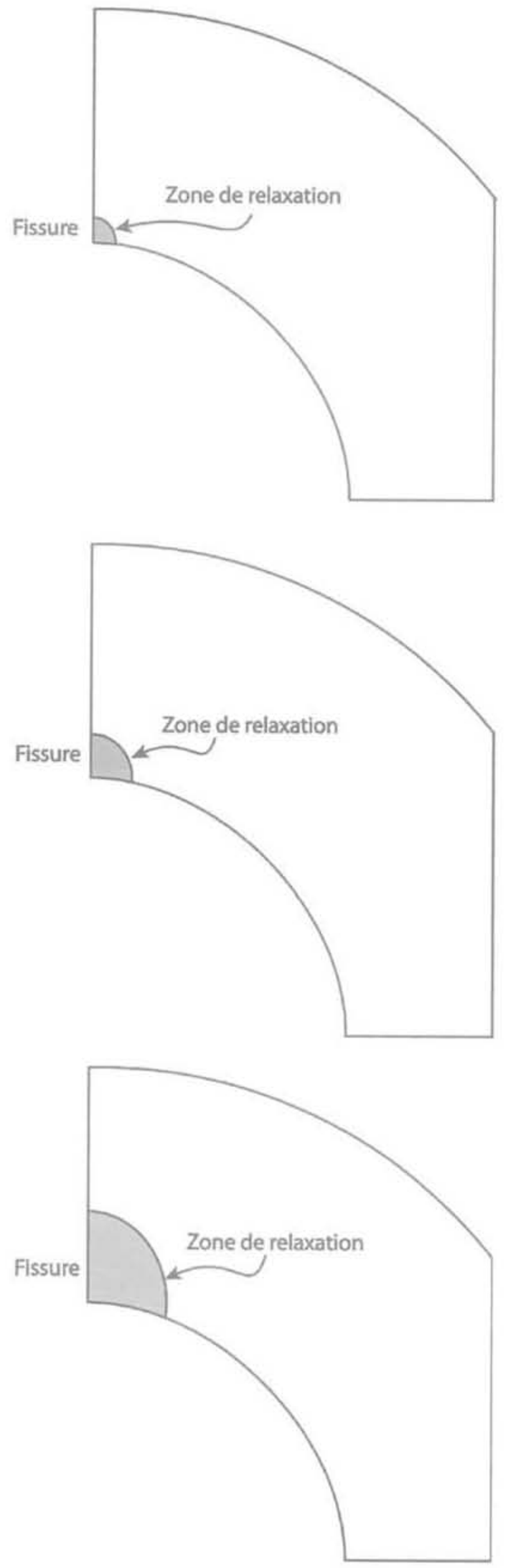

Fig.6 Évolution de la zone de relaxation des contraintes orthoradiales au voisinage d'une fissure. a) $\mathrm{a} / \mathrm{R}=0.1 ;$ b) $\mathrm{a} / \mathrm{R}=0.2 ; \mathrm{c}$ $\mathrm{a} / \mathrm{R}=0,4$.

Change of the obscuration zone around a crack for different sizes, a) $a / R=0.1 ; b) a / R=0.2$; c) $\mathrm{a} / \mathrm{R}=0.4$. importante et $\lambda_{b}(p) \ll \lambda_{t}(p)$. Cette différence peut conduire à une saturation de la fissuration.

On définit une densité caractéristique $\lambda$ et une pression caractéristique $\mathrm{p}_{\mathrm{f}}$ comme suit :

$$
\lambda_{c}=\lambda_{0}\left(\frac{p_{c}}{\sigma_{0}}\right)^{m} \text { et } p_{c}^{m+n}=\frac{\sigma_{0}^{m+n}}{\lambda_{0} A^{n}}
$$

La pression caractéristique correspond à un niveau de pression pour lequel $\lambda_{r}\left(p_{c}\right) Z_{\text {obs }}\left(p_{f}\right)=1$. En utilisant ces quantités caractéristiques, une solution analytique est obtenue pour l'équation différentielle (18):

$$
\frac{\lambda}{\lambda_{c}}=\left(\frac{m+n}{m}\right)^{\frac{-n}{m+n}} \gamma\left[\frac{m}{m+n} ; \frac{m}{m+n}\left(\frac{p}{p_{c}}\right)^{m+n}\right]
$$

où $\gamma$ est la fonction de gamma incomplète et l'indice c désigne les quantités caractéristiques définies plus haut. La figure 7 montre le phénomène de saturation du réseau de fissures et la variation de probabilité d'occultation quand la pression interne dépasse la pression caractéristique. La densité de fissuration à saturation s'obtient de l'équation (21) et ne dépend que du module de Weibull m (I'hétérogénéité de la roche) et de la dimension de l'espace $n$ quand elle est normée par $\lambda$.

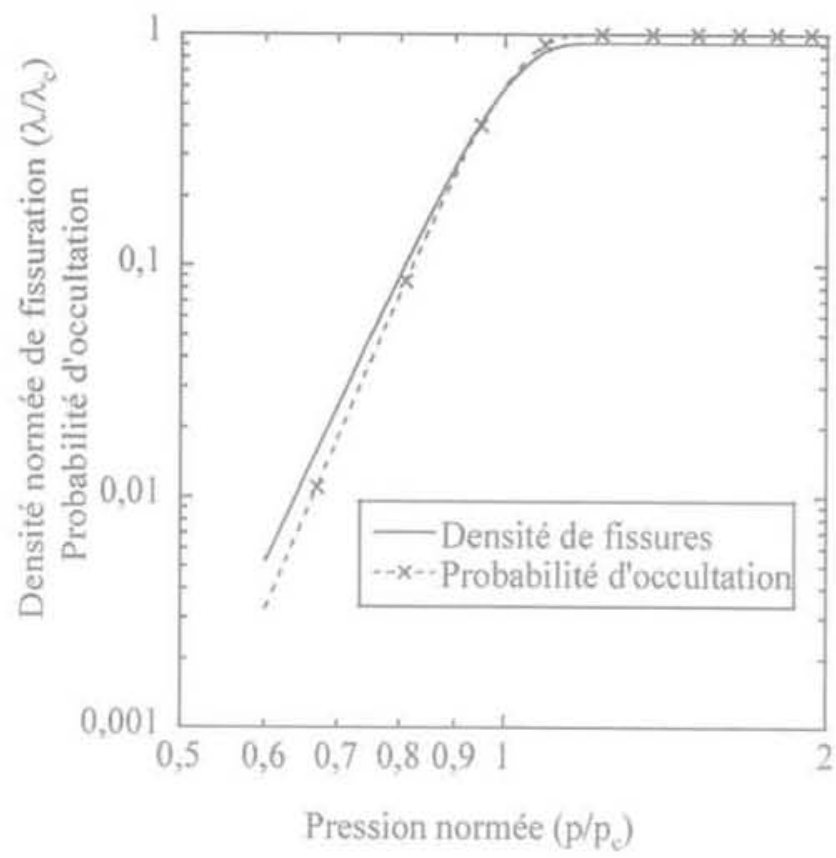

FiG.7 Probabilité d'occultation et densité normée de fissuration en fonction de la pression appliquée $(m=10, n=1)$.

Obscuration probability and normalized crack density as functions of the normalized applied presure $(m=10, n=1)$.

La figure 8 montre l'effet du module de Weibull sur la densité de físsuration. Avec un grand module de Weibull, la densité de fissuration augmente pendant un petit incrément de pression d'une manière importante quand la pression appliquée dépasse la valeur $p_{c}$. A cause de la dépendance du mécanisme de saturation à la pression appliquée, plus de fissures peuvent s'amorcer avant une saturation significative et le matériau sera complètement fissuré. A contrario, pour un petit module de Weibull $\mathrm{m}$, il y a plus de temps entre l'amorçage de deux fissures. Les premières fissures initiées écrantent les autres sites avant qu'ils ne puissent initier de nouvelles fissures. 


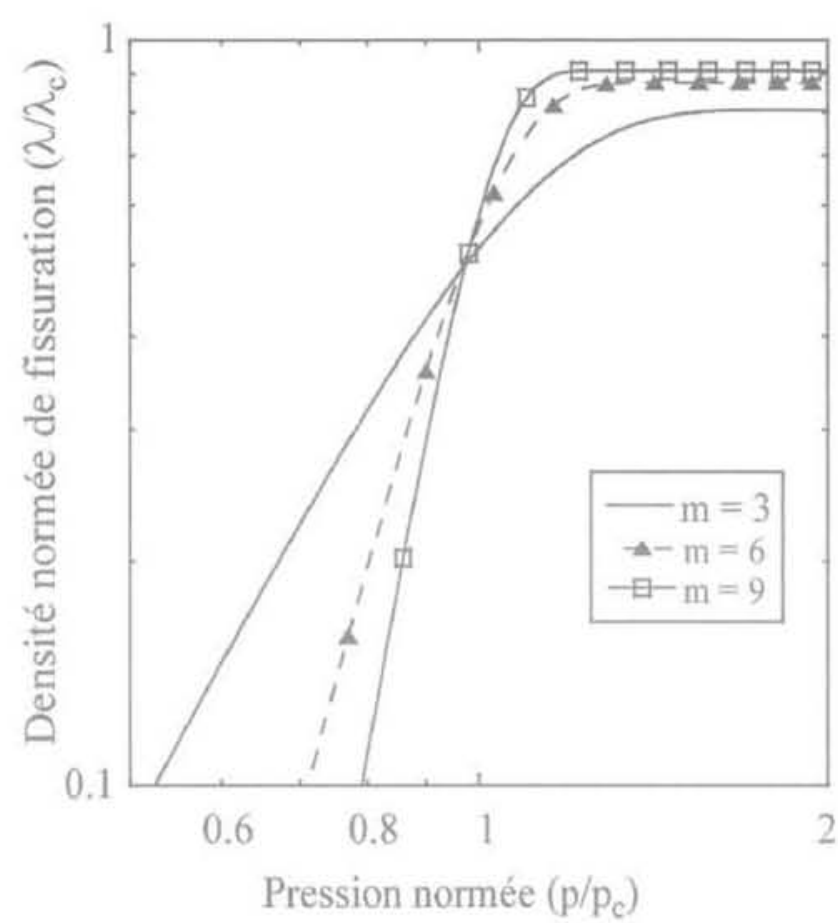

AG.8 Effet du module de Weibull sur la densité de fissuration $(n=1)$.

Effect of the Weibull modulus $m$ on the cracking density versus applied pressure $(n=1)$.

\section{Conclusion}

La détermination de la pression limite d'injection ainsi que l'étude des conséquences si jamais la pression d'injection dépasse cette valeur limite sont des parties intégrantes de l'étude de sûreté d'un site de stockage géologique de $\mathrm{CO}_{2}$. Une approche probabiliste de formation de réseaux de fractures prenant en compte l'effet de l'hétérogénéité de la roche est proposée dans cette optique.

En étudiant la propagation d'une fissure isolée, la possibilité de formation d'un réseau de fissures autour d'un puits d'injection ou à l'interface réservoir - couverture est démontrée. Un modèle probabiliste basé sur une distribution aléatoire de défauts initiaux, considérés comme les sites potentiels d'amorçage, a été proposé pour décrire les différents aspects de la formation de réseaux de fissures suite à l'injection de $\mathrm{CO}_{2}$. La densité totale de défauts est modélisée par un processus ponctuel de Poisson et l'interaction entre les fissures est prise en compte par la probabilité d'écrantage (ou d'obscurcissement). Une solution analytique a été obtenue pour calculer la probabilité d'écrantage dans le réseau ainsi que la densité de fissuration. L'extension de ce travail pour prendre en compte des couplages hydromécaniques et l'effet de la fracturation sur la perméabilité des formations de couverture en considérant des géométries plus réalistes est en cours.

\section{REMERCIEMENTS} Ce
BRGM

Ce travail a été financé par la Direction de la recherche du 
Budiansky B., O'Connell R.J. - Elastic Moduli of a Cracked System. Int. J. Solids Struct. 12, 1976, p. 81-97.

Coleman B.D. - Statistics and Time-Dependence of Mechanical Breakdown in Fibers. J. Appl. Phys. 29, 1958, p. 968 983.

Cox H. Heedrick J.P. Meer L.G. Straaten R.V.D. Holloway S, - The underground disposal of carbon djoxide. In Safety and stability of underground $\mathrm{CO}_{2}$ storage, Final report of the Joule If project $n^{\circ}$ CT192-0031. S. Holloway (Eds) 1996

Dangla P. Coussy O. - Non-linear poroelasticity for unsaturated porous materials: an energy appraoch. Poromecha nics, A tribute to M.A. Biot. Proc. of the Biot conference on poromechanics, Balkema, 1998.

Daniels H.E. - The Statistical Theory of the Strength of Bundles of Threads. Proc. $R$. Soc. London, A 183, 1944, p. 405-429

Denoual C. Hild F. - A Damage Model for the Dynamic Fragmentation of Brittle Solids. Comp. Meth. Appl. Mech. Eng. 183, 2000, p. 247-258.

Farrer C.D., Sorey W.C., Evans J.F., Howle B.D., Kerr B.M. - Forest-killing diffuse $\mathrm{CO}$, emissions at Mammoth Mountain as a sign of magnetic unrest. Nature 376, 1995, p. 675-677.

Freudenthal A.M. - Statistical Approach to Brittle Fracture: Fracture, H. Liebowitz (Ed.). New York (USA), Academic Press. 2. 1968, p. 591-619.

Gulino R., Phoenix S.L. - Weibull Strength Statistics for Graphite Fibres Measured from the Break Progression in a Model Graphite/Glass/Epoxy Microcomposite. J. Mater. Sci. 26 (11), 1991, p. 3107-3118.

Halm D., Dragon A. - A model of anisotropic damage by meso-crack growth; unilateral effect. Int. J. of Damage Mechanics 5, 1996, p. 384-402.
Hashin Z. - A differential scheme and its application to cracked materials. J. Mech. Phys. Solids 36, 1988, p. 719-734.

Hild F. - The Weibull law: a model of wide applicability. Physical Aspects of Fracture, E. Bouchaud, D. Jeulin, C. Prioul et S. Roux (Eds.), Kluwer Academic Publishers, 2001, p. 35-46.

Hild F., Billardon R., Marquis D, - Hétérogénéité des contraintes et rupture des matériaux fragiles. C.R. Acad. Sci. Paris t. 315 (Série II), 1992, p. 1293-1298

Hild F. Domerque IM. Fvans A G L Leckie F.A. - Tensile and Flexural Ultimate Strength of Fiber-Reinforced CeramicMatrix Composites, Int. J. Solids Struct. 31 (7). 1994, p. $1035-1045$.

Hild F. Marquis D. - Fiabilité de matériaux avec défauts en propagation stable. C.R Acad. Sci. Paris t. 320 (Série IIb), 1995. p. $57-62$

Holloway S. - Underground sequestration of carbon dioxide-a viable greenhouse gas mitigation option. Energy 30, 2005, p. $2318-2333$.

Homand-Étienne F. Hoxa D. Shao J.F. - A continium damage law for brittle rocks. Computers and Geotechnics 22 (2), 1998, p. 135-151.

Hoxa D. Homand F. - Modèlisation microstructurale de l'endommagement des roches fragiles. Congrès français de mécanique, Toulouse, 1999.

Jeullin D. - Modèles morphologiques de structures aléatoires et changement d'échelle. Thèse d'État, université de Caen, 1991.

Kachanov L.M. - Time of the Rupture Process under Creep Conditions. Bull. SSE Acad. Sci. Division of Technical Sciences 8, 1958, p. 26-31.

Kachanov M. - Elastic Solids with Manv Cracks and Related Problems. Adv. Appl. Mech. 30, 1994, p. 259-445.
Le Guren F. Sigvaldason G.E. - The Lake Nyos event and natural $\mathrm{CO}$, degassing. J. Volcanol Geotherm Res, 39, 1989, p. 95-276.

Lemaitre J., Chaboche J.L. - Aspect phénoménologique de la rupture par endommagement. J. Méc. Appl. 2 (3) 1978, p. 317-365.

Lemaitre J., Chaboche J.L. - Mécanique des matériaux solides. Dunod, 1985

Rutqvist J., Tsang C.F, - Coupled hydromechanical effects in $\mathrm{CO}_{2}$ injection. Lawrence Berkeley National Laboratory report (http://www-library.Jbl.gov/docs LBNL/573/37/PDF/LBNL-57337.pdif 2005.

Rutqvist I., Wu Y.S., Tsang C.F., Bodvarsson G. - A modeling approach for analysis of coupled multiphase fluid flow. heat transfer and deformation in fractured porous rock. Int. J. Rock Mech. \& Min. Sci. 39, 2002, p. 429-442.

Shao J.F., Hoxha D. Bart M. Homand F. Duveau G., Souly M., Hoteit N. - Modelling of induced anisotropic damage in granites. Int. J. Rock Mech. \& Min. Sci. 36. 1999, p. 1001-1012.

Souley M. Homand F., Pepa S., Hoxha D. Damage-induced permeability changes in granite: a case example at the URL in Canda. Int. J. Rock Mech. \& Min. Sci. 38 , 2001, p. 297-310

Weibull W. - A Statistical Theory of the Strength of Materials 151 Roy. Swed. Inst. Eng. Res., 1939.

Weibull W. - A Statistical Distribution Function of Wide Applicability. ASME I Appl. Mech. 18 (3). 1951, p. 293-297.

Williams S.N. - Dead trees tell tales. Nature $376,1995, \mathrm{p}, 644$ 